\title{
Evaluation of the Pulse Pressure Index at the Peak of Exercise Before and After Cardiac Rehabilitation
}

\author{
Amir Hossein Yazdi ${ }^{1}$, Parnian Kazemi ${ }^{2}$, Mehrnaz Olfat ${ }^{2}$, \\ Farzaneh Esna-Ashari ${ }^{3}$, Leila Najmafshar ${ }^{1,}{ }^{*}$ \\ ${ }^{1}$ Cardiology Department, School of Medicine, Hamadan University of Medical \\ Sciences, Hamedan, Iran \\ ${ }^{2}$ School of Medicine, Hamadan University of Medical Sciences, Hamedan, Iran \\ ${ }^{3}$ Epidemiology Department, School of Medicine, Hamadan University of Medical \\ Sciences, Hamedan, Iran \\ * Corresponding author: Leila Najmafshar, Cardiology Department, School of \\ Medicine, Hamadan University of Medical Sciences, Hamedan, Iran. E-mail: Leila. \\ najmafshar@gmail.com
}

DOI: $10.21859 /$ ijcp-020303

Submited: 03.06.2017

Accepted: 06.01.2017

Keywords:

Rehabilitation

Coronary Vessels

Coronary Artery Bypass

(C) 2017. International Journal of

Cardiovascular Practice.

\begin{abstract}
Introduction: As a new supplementary therapeutic option, cardiac rehabilitation (CR) is getting more attention every day. Several studies have proved its positive impact on qualitative criteria; but few studies have been done on quantitative criteria. The purpose of the present study is to evaluate the impact of CR on non-invasive measurement of maximum cardiac output by Pulse Pressure index (PPI) at the peak of exercise.

Methods: This is a non-randomized prospective cohort study conducted in Hamadan, Iran in 2015. One hundred eligible patients who had undergone coronary artery bypass surgery participated in our study, after obtaining cardiologist permission and informed consent. The PPI was measured at the peak of exercise before and after standard CR program.

Results: Mean PPI was not significantly different before and after CR. PPI was significantly increased in patients younger than 60 years old $(\mathrm{P}=0.022)$. In contrast to hypertensive patients, PPI increased significantly after CR in non-hypertensive patients $(\mathrm{P}=0.002)$. PPI was significantly increased in non-diabetic patients after $\mathrm{CR}(\mathrm{P}=$ 0.046), but not in diabetic individuals. Other variables did not show any statistically significant effect on PPI in response to CR.

Conclusions: Findings from our study revealed that PPI is associated with vascular atherosclerosis, as well as cardiac output; Positive effects of CR diminish in older, diabetic and hypertensive patients with more progressive atherosclerosis.
\end{abstract}

\section{INTRODUCTION}

Cardiovascular events, particularly coronary artery diseases $(\mathrm{CAD})$, are the most common cause of death worldwide [1]; accounting for almost half of the deaths in industrialized countries and 25 percent in developing ones. These staggering numbers are due to the disease called coronary arteries atherosclerosis which results in impaired myocardial blood supply and subsequent ischemia and infarction [2]. The most important risk factors are male gender, age, positive family history of CAD, smoking, immobility and obesity, hypertension (HTN), hyperlipidemia (HLP), diabetes mellitus (DM), and metabolic syndrome [3]. In general, therapeutic options consist of life style modification, medical therapy and revascularization methods including percutaneous coronary intervention (PCI) and coronary artery bypass graft (CABG).

Cardiac rehabilitation (CR) is a medically supervised program which helps improve patients' health and speed up their physical and psychological recovery from acute cardiovascular events or coronary artery bypass graft [4-7]. CR Programs usually involve patient assessment including medical history (evaluation of comorbidities, risk factors, and medications); physical examination; nutritional counseling and weight management; smoking cessation; psychological management of anger, anxiety and depression; physical activity counseling and exercise training. One of the primary steps before participation in CR is exercise tolerance test (ETT), so that the individuals' exercise recommendations will be designed based on the findings of the test, which results in best specification of duration and severity of physical activity [8]. Pulse pressure index (PPI) is an indirect indicator of cardiac output and a good predictor for cardiovascular complications. It is defined as the pulse pressure (systolic blood pressure - diastolic blood pressure) divided by systolic blood pressure (SBP) [9]. Since cardiovascular events are increasv 
ing quickly among human societies resulting in major disabilities, and on the other hand CR programs improves life quality and reduces complications of surgery, and also the point that most surveys have studied the effects of CR on life quality, it is necessary to study the effects of CR on myocardial function by evaluation cardiac function index. The goal of this study is a comparison between PPI before and after 12 sessions of $\mathrm{CR}$ in patients undergoing CABG.

\section{METHODS}

This is a non-randomized prospective cohort study (before and after study) conducted in Hamadan, Iran in 2015. The study protocol was approved by the institutional ethic committee of Hamadan University of Medical Sciences. After signing the informed consent forms and obtaining cardiologist permission, 100 eligible patients entered the study. Exclusion criteria were unwillingness of the patient to participate in the program or any condition prohibiting the patient from exercise, i.e. musculoskeletal deformities, major acquired disabilities and severe left ventricle (LV) dysfunction. Before and after participation in 12 sessions of CR with standard protocol, patients underwent ETT with standard Bruce protocol. PPI was calculated at the peak of exercise for each patient before initiation and also at the end of CR program. For all participants, blood pressure and index measurements were performed by the same health professional and one specified standardized device. All patient were treated by standard medical and nutritional protocol and medications were continued before ETTs. For each patient, a questionnaire of needed information (our variables) was filled either by direct questioning or data extraction from their medical records. Data gathered from the questionnaires were analyzed by SPSS version 16 software. Statistical analysis was performed using Paired t-test and signed-rank test. P value < 0.05 was considered significant.

\section{RESULTS}

One hundred patients enrolled in this study including 63 (63\%) men and 37 (37\%) women. Mean age of participants was $60 \pm 5$ years with minimum age of 44 years old and maximum age of 80 years old. Patients' demographics are summarized in Table 1.

\begin{tabular}{|lc|}
\hline Table 1: Patient Characteristics & \\
Characteristics & $\mathrm{N}=100$ \\
Age (years) & $60 \pm 5$ \\
Female & $37(37 \%)$ \\
Diabetes mellitus & $36(36 \%)$ \\
Hypertension & $50(50 \%)$ \\
hyperlipidemia & $50(50 \%)$ \\
Smoker & $33(33 \%)$ \\
Left ventricular function & \\
$\quad \mathrm{EF}>=45 \%$ & $84(84 \%)$ \\
$\quad 30 \%<\mathrm{EF}<45 \%$ & $16(16 \%)$ \\
$\mathrm{EF}<=30 \%$ & $0(0 \%)$ \\
\hline
\end{tabular}

Data in table are presented as No. (\%) or Mean \pm SD

Abbreviations: EF, ejection fraction

\begin{tabular}{|c|c|c|c|c|c|}
\hline PPI changes in different groups & Number & PPI before CR & PPI after CR & Mean Difference & Pvalue \\
\hline \multicolumn{6}{|l|}{ Gender } \\
\hline Male & 63 & $0.34 \pm 0.04$ & $0.35 \pm 0.04$ & $0.007 \pm 0.05$ & 0.22 \\
\hline Female & 37 & $0.36 \pm 0.05$ & $0.37 \pm 0.05$ & $0.011 \pm 0.06$ & 0.02 \\
\hline \multicolumn{6}{|l|}{ Age } \\
\hline$<60$ years & 48 & $0.33 \pm 0.04$ & $0.36 \pm 0.04$ & $0.01 \pm 05$ & 0.022 \\
\hline$>=60$ years & 52 & $0.35 \pm 0.01$ & $0.35 \pm 0.04$ & $0.002 \pm 06$ & 0.52 \\
\hline \multicolumn{6}{|l|}{$\mathbf{D M}$} \\
\hline Yes & 36 & $0.37 \pm 0.04$ & $0.37 \pm 0.05$ & $0.005 \pm 0.05$ & 0.60 \\
\hline No & 64 & $0.34 \pm 0.04$ & $0.35 \pm 0.04$ & $0.01 \pm 0.05$ & 0.046 \\
\hline \multicolumn{6}{|l|}{ DLP } \\
\hline Yes & 50 & $0.36 \pm 0.04$ & $0.36 \pm 0.05$ & $0.001 \pm 0.06$ & 0.24 \\
\hline No & 50 & $0.34 \pm 0.04$ & $0.35 \pm 0.04$ & $0.008 \pm 0.05$ & 0.091 \\
\hline \multicolumn{6}{|l|}{ HTN } \\
\hline Yes & 50 & $0.36 \pm 0.04$ & $0.36 \pm 0.04$ & $0.001 \pm 0.06$ & 0.92 \\
\hline No & 50 & $0.33 \pm 0.04$ & $0.35 \pm 0.04$ & $0.01 \pm 0.05$ & 0.002 \\
\hline \multicolumn{6}{|l|}{ Smoker } \\
\hline Yes & 33 & $0.33 \pm 0.04$ & $0.34 \pm 0.04$ & $0.01 \pm 0.05$ & 0.10 \\
\hline NO & 67 & $0.35 \pm 0.04$ & $0.36 \pm 0.05$ & $0.01 \pm 0.06$ & 0.18 \\
\hline \multicolumn{6}{|l|}{ LV function } \\
\hline Moderate LV dysfunction & 16 & $0.34 \pm 0.02$ & $0.35 \pm 0.04$ & $0.01 \pm 0.04$ & 0.25 \\
\hline Relatively normal LV function & 84 & $0.35 \pm 0.04$ & $0.36 \pm 0.04$ & $0.01 \pm 0.06$ & 0.21 \\
\hline
\end{tabular}

Data in table are presented as No. (\%) or Mean \pm SD

Abbreviations: PPI, Pulse Pressure index; EF, ejection fraction; DM, diabetes mellitus; DLP, dyslipidemia; HTN, hypertension; LV, left ventricle; $\mathrm{CR}$, cardiac rehabilitation 
The minimum and maximum of measured peak SBP before $\mathrm{CR}$, was 90 and $155 \mathrm{mmHg}$ respectively. The mean of peak $\mathrm{SBP}$ was $121.95 \pm 14.21 \mathrm{mmHg}$. The minimum and maximum of measured SBP after CR, was $100 \mathrm{mmHg}$ and 150 $\mathrm{mmHg}$ respectively with mean of $121.55 \pm 11.84 \mathrm{mmHg}$. Mean of SBP did not significantly change after CR $(\mathrm{P}=0.76)$. The minimum of measured DBP before CR was $60 \mathrm{mmHg}$ and the maximum was $90 \mathrm{mmHg}$ with mean of $78.55 \pm 7.18$ mmHg. The minimum of measured DBP after CR was 60 $\mathrm{mmHg}$ and maximum was $90 \mathrm{mmHg}$. The mean DBP was $77.35 \pm 7.08 \mathrm{mmHg}$. Mean of DBP was not significantly different before and after CR $(P=0.12)$ Minimum of PPI before CR was 0.33 and maximum of it was 0.83 with mean of $0.35 \pm 0.046$. After $C R$, minimum of PPI was 0.27 and maximum was 0.53 with mean of $0.36 \pm 0.048$. Mean of PPI was not significantly different before and after CR $(\mathrm{P}=0.12)$. PPI changes were studied separately in different groups (Table 2): PPI did not significantly increase before and after CR, neither in males nor in females. PPI in patients younger than 60 years old significantly increased after $\mathrm{CR}(\mathrm{P}=0.022)$. But $\mathrm{CR}$ in patients older than this age didn't have any significant effect on PPI.PPI among diabetic patients was not significantly different before and after CR $(P=0.060)$; however, it increased in non-diabetic individuals $(\mathrm{P}=0.046)$. PPI was not statistically different before and after CR neither in patients with HLP nor in patients with normal lipid profile. PPI among hypertensive patients was not significantly different before and after CR; however, it increased in non-hypertensive individuals after $C R(P=0.002)$. $C R$ had no significant effect on PPI in smokers and non-smoker patients. According to LV dysfunction degree, PPI in patients did not change after CR.

\section{DISCUSSION}

We studied 100 patients, including 63 males and 37 females. In overall, we did not find out any significant difference between mean of PPI before and after CR. Based on world health organization (WHO) reports, more than 17 million people lose their lives because of the cardiovascular events annually and CVD is proposed as the most important disease and the main cause of death by year 2020 due to high mortality and disability rate and also its imposed economic burden [10]. PPI is an indirect quantitative predictor of cart diac output and is defined as the pulse pressure divided by SBP [11]. On the other hand, one of the best measures to increase $\mathrm{CABG}$ effectiveness is $\mathrm{CR}$. The goal of this study was to evaluate the effect of CR on PPI in patients undergoing CABG. In Mc Kee et al survey in 2007, the incidence of CVD in adults had increased after third decade of life and the maximum was between age of 45 to 75 years old [12]. In the present study, peak SBP was not significantly affected by CR. Fang cui et al in 2012 studied the effect of CR on exercise tolerance after PCI and demonstrated that peak SBP was not different before and after CR [13]. In a survey done by Siavashi et al in 2012, the effect of CR on hemodynamic status after CABG were evaluated, SBP of patients after CR was not significantly different in comparison with the control group [14]. These results are accordant with findings from the present study.

In our study, the DBP was not significantly different before and after CR, like the results reported by Ghalamghash et al study in 2008 and Siavashi et al study in 2012 [14]. In conz trast, in the survey done by Steki et al in 2011, two-monthslong CR significantly improved functional capacity and some hemodynamic responses such as resting and maximum systolic and diastolic blood pressure, resting and maximum heart rate, ejection fraction, and rate pressure product in post CABG patients [15]. In our study, 12 sessions of CR did not affect the PPI. Cai et al in 2015 showed that PPI have a significant relationship with intima-media carotid thickness in adults and thus it is a valuable parameter for primary screening of patients with atherosclerosis risk [16]. We can conclude that PPI is associated with vascular atherosclerosis, as well as cardiac output; as a result it's expected that PPI cannot completely show the effect of CR on cardiac function alone; and more accurate indicators of cardiac output like angiographic calculations or cardiac output measurements by the Fick method that effects on cardiac function are needed, but because of their risks and invasive nature of the procedures, they are not routinely performed for this purpose. In our study, PPI was significantly increased in patients younger than 60 years old. Since aging results in cardiac fibrosis and increased myocardium scar tissue, cardiac reserve is reduced even after CABG and CR. So positive effects of CR are more pronounced in younger patients [17].

In non-hypertensive patients, PPI is increased significantly after CR, in contrast to hypertensive patients. Since HTN has known effects on myocardium by LV hypertrophy, rate of increased cardiac reserve during exercise in hypertensive patients is less than non-hypertensive ones [18]. PPI is signifih cantly increased in non-diabetic patients after CR, but not in diabetic individuals. Verges et al in 2004 reported that exercise compliance of type 2 diabetic patients after an ischemic event, is significantly lower and blood glucose level influences on response to CR in diabetic patients[19]. In our study, gender, HLP, smoking and LV dysfunction severity had not any significant effect on PPI in response to CR.

\section{CONCLUSIONS}

The goal of present study was to compare PPI before and after $\mathrm{CR}$ in post $\mathrm{CABG}$ patients. Although the results showed that PPI didn't have any significant increase after 12 sessions of CR; However, Positive effects of CR diminish in older, diabetic and hypertensive patients with more progressive atherosclerosis.

\section{AUTHORS' CONTRIBUTION}

Data collection: Kazemi, Olfat; Data analysis and interpretation: Esna-Ashari; Drafting the article:Yazdi; Critical revision of the article:Yazdi, Najmafshar; Final approval of the version to be published:Yazdi, Najmafshar

\section{CONFLICTS OF INTERESTS}

There is no conflict of interest.

\section{FUNDING}

Authors declared there is no funding or support. 


\section{REFERENCES}

1. Antman E, Selwyn A, Braunwald E, Loscalzo J. Ischemic heart disease. In: Fauci A, Kasper D, Hauser S, Longo D, Jameson J, Loscalzo J, editors. Harrison's Internal Medicine. 1. 18th ed. New York: Mac Graw Hill; 2011. p. 13.

2. Andreoli T, Benjamin L, Griggs R, Wing E. Cecil Essential Of Medicine 8th edition. ed. Philadelphia: Saunders Elsevier; 2010. 23 p. Bonow R, Mann D, Zipes D, libby P. ST-Segment Elevation myocardial Infarction:Pathology,Pathophysiology,and Clinical Features. 9th edition. ed. Philadelphia:: Saunders Elsevier; 2012. 24 p.

4. Suaya JA, Shepard DS, Normand SL, Ades PA, Prottas J, Stason WB. Use of cardiac rehabilitation by Medicare beneficiaries after myocardial infarction or coronary bypass surgery. Circulation. 2007;116(15):1653-62. DOI: 10.1161/CIRCULATIONAHA.107.701466 PMID: 17893274

5. Martin BJ, Hauer T, Arena R, Austford LD, Galbraith PD, Lewin $\mathrm{AM}$, et al. Cardiac rehabilitation attendance and outcomes in coronary artery disease patients. Circulation. 2012;126(6):677-87. DOI: $\underline{10.1161 / C I R C U L A T I O N A H A .111 .066738}$ PMID: 22777176

6. Khalife-Zadeh A, Dorri S, Shafiee S. The effect of cardiac rehabilitation on quality of life in patients with acute coronary syndrome. Iran J Nurs Midwifery Res. 2015;20(5):588-93. DOI: 10.4103/17359066.164504 PMID: 26457097

7. Masoumi SZ, Kazemi F, Khani S, Seifpanahi-Shabani H, Garousian M, Ghabeshi M, et al. Evaluating the Effect of Cardiac Rehabilitation Care Plan on Quality of Life of Patients Undergoing Coronary Artery Bypass Graft Surgery. Int J Cardiovasc Pract. 2017;2(2):1-7. DOI: $10.21859 /$ ijcp-020204

8. Balady GJ, Ades PA, Comoss P, Limacher M, Pina IL, Southard D, et al. Core components of cardiac rehabilitation/secondary prevention programs: A statement for healthcare professionals from the American Heart Association and the American Association of Cardiovascular and Pulmonary Rehabilitation Writing Group. Circulation. 2000;102(9):1069-73. PMID: 10961975

9. Peng-Lin Y, Yue-Chun L. Pulse pressure index (pulse pressure/systolic pressure) may be better than pulse pressure for assessment of cardiovascular outcomes. Med Hypotheses. 2009;72(6):729-31. DOI: 10.1016/j.mehy.2008.12.041 PMID: 19231092
10. Gillespie CD, Wigington C, Hong Y, Centers for Disease C, Prevention. Coronary heart disease and stroke deaths - United States, 2009. MMWR Suppl. 2013;62(3):157-60. PMID: 24264507

11. Safar ME, Levy BI, Struijker-Boudier H. Current perspectives on arterial stiffness and pulse pressure in hypertension and cardiovascular diseases. Circulation. 2003;107(22):2864-9. DOI: 10.1161/01. CIR.0000069826.36125.B4 PMID: 12796414

12. McKee G, Bannon J, Kerins M, FitzGerald G. Changes in diet, exercise and stress behaviours using the stages of change model in cardiac rehabilitation patients. Eur J Cardiovasc Nurs. 2007;6(3):233-40. DOI: 10.1016/J.EJCNURSE.2006.10.002 PMID: 17158092

13. Cui F, Ren Y, Jin H, Cui B. Rehabilitation training improves exer cise tolerance after percutaneous coronary intervention. J Biomed Res. 2012;26(4):248-52. DOI: 10.7555/JBR.26.20110119 PMID: 23554756

14. Siavoshi S, Roshandel M, Zareiyan A, Etefagh L. The effect of cardiac rehabilitation on hemodynamic status in patients after coronary artery bypass surgery. Evid Based Care. 2013;2(4):19-27.

15. Ghashghaei FE, Sadeghi M, Marandi SM, Ghashghaei SE. Exercise-based cardiac rehabilitation improves hemodynamic responses after coronary artery bypass graft surgery. ARYA Atheroscler. 2012;7(4):151-6. PMID: 23205048

16. Cai A, Mo Y, Zhang Y, Li J, Chen J, Zhou Y, et al. Relationship of pulse pressure index and carotid intima-media thickness in hypertensive adults. Clin Exp Hypertens. 2015;37(4):267-70. DOI: 10.3109/10641963.2014.954713 PMID: 25375964

17. Lakatta EG, Levy D. Arterial and cardiac aging: major shareholders in cardiovascular disease enterprises: Part II: the aging heart in health: links to heart disease. Circulation. 2003;107(2):346-54. DOI: 10.1161/01.cir.0000048893.62841.f7 PMID: 12538439

18. Brilla CG, Janicki JS, Weber KT. Impaired diastolic function and coronary reserve in genetic hypertension. Role of interstitial fibrosis and medial thickening of intramyocardial coronary arteries. Circ Res. 1991;69(1):107-15. PMID: 1647274

19. Verges B, Patois-Verges B, Cohen M, Lucas B, Galland-Jos C, Casillas JM. Effects of cardiac rehabilitation on exercise capacity in Type 2 diabetic patients with coronary artery disease. Diabet Med. 2004;21(8):889-95. DOI: 10.1111/i.1464-5491.2004.01262.x PMID: 15270793 